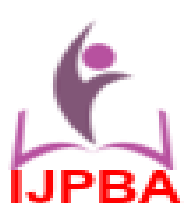

Contents lists available at www.ijpba.in

International Journal of Pharmaceutical and Biological Science Archive

PubMed (National Library of Medicine ID: 101738825)

Index Copernicus Value 2017: 71.80

Volume 7 Issue 2; March-April; 2019; Page No. 247-250

\title{
A REVIEW ON COMPARATIVE STUDY ON THE SAFETY AND EFFECTIVENESS OF SACUBITRIL- VALSARTAN COMBINATION WITH ACE/ARB THERAPY IN CARDIAC PATIENTS
}

\author{
Bhagya Suresh $^{1 *}$, Mathew George ${ }^{2}$, Lincy Joseph ${ }^{3}$ \\ ${ }^{1}$ Department of Pharmacy Practice, Pushpagiri College of Pharmacy, Thiruvalla-689107, Kerala, \\ India. \\ ${ }^{2}$ Department of Pharmacology, Pushpagiri College of Pharmacy, Thiruvalla-689107, Kerala, India. \\ ${ }^{3}$ Department of Pharmaceutical Chemistry, Pushpagiri College of Pharmacy, Thiruvalla-689107, \\ Kerala, India.
}

\begin{abstract}
Cardiovascular (CV) disease is a major cause of morbidity and mortality in the developing and the developed world, and represents a major barrier to sustainable human development. Ischemic heart disease, cerebrovascular disease, cardiomyopathy and heart failure (HF), and hypertension among others represent major forms of CV disease. Heart failure (HF) is among the key contributors to the CV-related health care burden, a uninterrupted concern despite the utilization of clinically tried guideline-directed therapies. The most common cause for HF is reduced left cavum heart muscle perform. ARBs produce equivalent mortality benefits with fewer adverse effects than ACE inhibitors. Angiotensin converting enzyme (ACEI) reduces the combined risk of death or hospitalization, slow progression of $\mathrm{HF}$, and reduced rate of reinfarction. Sacubitril/valsartan could be a first-in-class twin action molecule of the neprilysin (NEP) substance sacubitril (AHU-377) and therefore the angiotensin II (Ang II) sort one (AT1) receptor blocker (ARB) valsartan. The beneficial antihypertensive and HF effects of sacubitril/valsartan are mediated through the inhibition of NEP in catabolizing the natriuretic peptides (NPS) and the blockade of Ang II, AT1 receptor with valsartan. These actions of sacubitril/ valsartan end in general dilation and inflated symptoms and symptoms, resulting in decrease in peripheral tube resistance and plasma volume contraction, all necessary actions for the lowering of BP and improving HF symptoms.
\end{abstract}

Keywords: cardiovascular disease, left ventricular ejection fraction, angiotensin II receptor blocker, angiotensin converting enzyme, sacubitril/valsartan.

\section{INTRODUCTION}

Cardiovascular (CV) disease is a major cause of morbidity and mortality in the developing and the developed world, and represents a major barrier to sustainable human development. Ischemic heart disease, cerebrovascular disease, cardiomyopathy and heart failure (HF), and hypertension among others represent major forms of CV disease. Mortality from CV sickness had plateaued within the recent years rising regarding concerning alarms about the sustained effectivity of obtainable preventive and treatment choices. Heart failure (HF) is among the key contributors to the CV-related health care burden, a dogging concern despite the utilization of clinically verified guidelinedirected therapies ${ }^{[1]}$.

Heart failure is a clinical syndrome caused by structural and functional defects in myocardium resulting in impairment of ventricular filling or the ejection of blood. The most common cause for HF is reduced left cavum heart muscle perform ${ }^{[2]}$. The incidence and prevalence estimates of $\mathrm{HF}$ are unreliable in India because of the lack of surveillance systems to adequately capture these data ${ }^{[3]}$. $\mathrm{HF}$ is classified based on left ventricular ejection fraction (LVEF) into HF with reduced $\mathrm{EF}$ (HFrEF) with an LVEF $<40 \%$ and HF with preserved EF (HFpEF) with an LVEF $\geq 50 \%$. An EF 
between $40 \%$ and $49 \%$ is considered an intermediate zone and is termed as HF with borderline $\mathrm{EF}$ or $\mathrm{HF}$ with mid-range $\mathrm{EF}$. Epidemiologic data indicate that HFpEF and HFrEF contribute equally to the total HF population. HFpEF patients have a similar postdischarge mortality risk and equally high rates of re-hospitalization, compared to patients with $\mathrm{HFrEF}^{[4]}$.

Some of the major pathogenic mechanisms leading to $\mathrm{HF}$ are increased hemodynamic overload, ischemia-related dysfunction, ventricular remodeling, excessive neurohormonal stimulation, abnormal myocyte calcium cycling, accelerated apoptosis and genetic mutations.

The weakening of heart's pumping ability causes;

- Blood and fluid to back up into the lungs

- The buildup of fluid in the feet, angles and legs called edema

\section{- Tiredness and shortness of breath}

There are several medications to treat $\mathrm{CHF}$, including Angiotensin- Converting Enzyme inhibitor (enalapril, captopril), Angiotensin II Receptor Blockers (lorsatan, valsartan), betablocker (atenolol, bisoprolol), diuretics and surgery includes angioplasty. Angiotensin II receptor blockers (ARBs) block the angiotensin II receptor, preventing the deleterious effects of angiotensin II. ARBs produce equivalent mortality benefits with fewer adverse effects than ACE inhibitors. Angiotensin converting enzyme (ACEI) reduces the combined risk of death or hospitalization, slow progression of $\mathrm{HF}$, and reduced rate of reinfarction ${ }^{[2]}$. Advances in our understanding of the reninangiotensin-aldosterone (RAAS) pathway and natriuretic peptide system, lessons learned from randomized trials of natriuretic peptide system augmentation, and pharmacoinnovation led to the creation and validation of combination sacubitril/valsartan ([LCZ696]) for the treatment of $\mathrm{HFrEF}^{[4]}$.

Sacubitril/valsartan is a first-in-class dual action molecule of the neprilysin (NEP) inhibitor sacubitril (AHU-377) and the angiotensin II (Ang II) type 1 (AT1) receptor blocker (ARB) valsartan. The drug has been approved by both the Food and Drug Administration (FDA) and the European Medicines Agency (EMA) in 2015 for the treatment of heart failure with reduced ejection fraction [HFrEF] and awaits approval for the treatment of hypertension. The beneficial antihypertensive and HF effects of sacubitril/valsartan are mediated through the inhibition of NEP in catabolizing the natriuretic peptides (NPs) and the blockade of Ang II, AT1 receptor with valsartan. These actions of sacubitril/ valsartan result in systemic vasodilation and increased diuresis and natriuresis, leading to decrease in peripheral vascular resistance and plasma volume contraction, all important actions for the lowering of BP and improving HF symptoms ${ }^{[5]}$.

\section{REVIEW OF LITERATURE}

1) Ramesh R. Dargad et al [3] (2018) conducted a study in patients with hypertension who received sacubitril valsartan combination drug. Inclusion of the study was based mainly on the methods section of the trials. When obtainable, large, well- controlled trials with acceptable applied mathematics methodology was most popular. The study was conducted in 8442 adult patient with symptomatic chronic HF and systolic dysfunction. The study shows that sacubitril valsartan is more effective in improving symptoms and physical limitations, reducing the risk of cardiovascular death, HF hospitalization, and the overall mortality and morbidity compared to its counterparts.

2) Helena Norberg et al ${ }^{[6]}$ (2018), conducted a study to investigate the eligibility of the prospective comparison of angiotensin receptor- neprilysin inhibitor to determine impact on global mortality and morbidity in heart failure. A retrospective study in 2029 patients was performed, and 401 patients had ejection fraction of $<35 \% .95$ patients consummated all entering criteria and thus were eligible for sacubitril-valsartan. Only $24 \%$ of our real-world HFrEF population was eligible for sacubitril- valsartan, and the real world 
HFrEF patients were significantly older than the PARADIGM-HF population.

3) Oriol lborra-Egea et al ${ }^{[7]}$ (2017), the study was sought to explore the mechanistic details for sacubitril-valsartan in heart failure and post- myocardial infarction remodeling, using as in silico, systems biology approach. When analyzed independently, sacubitril was found to attenuate cardiomyocytes cell death, hypertrophy and impaired myocytes contractility by inhibiting PTEN. Valsartan improves cardiac remodeling by inhibiting the guanine nucleotides- binding protein family.

4) Victor shri et al ${ }^{[8]}$ (2017), the study was conducted to determine the clinical benefit for sacubitril-valsartan versus ACE inhibitor enalapril in patients with heart diseases with reduced ejection fraction. Patients were randomized to double daily sacubitril-valsartan $200 \mathrm{mg}$ or ACE inhibitors $10 \mathrm{mg}$. The study style incorporated 2 wash out periods ( $36 \mathrm{~h}$ each) to reduce any potential risk of angioedema thanks to overlapping ACE and neprilysin inhibition. Overall 148 suspected angioedema events, 54 were confirmed as angioedema by AAC. The frequency was higher in black patients. Most events were mild. There was no excess risk of angioedema with sacubitril-valsartan versus enalapril.

5) Yandrapalli S e tal ${ }^{[9]}$ (2017), conducted a Phase III double-blind controlled trial in 8442 patients with $\mathrm{LVEF}<40 \%$ who received twice daily dosing of either 200mg sacubitrilvalsartan or $10 \mathrm{mg}$ enalapril in addition to standard medical therapy for HF. At 27- month follow-up, LCZ696 was associated with $20 \%$ reduction in the composite primary end point death from CV causes or hospitalization for HF, compared to enalapril. Sacubitril - valsartan performed higher than ACE inhibitor across varied HFrEF patient characteristics and showed substantial profit in patients with different common co morbidities.

6) Randall.C.Starling ${ }^{[10]}$ (2015), conducted a study in the mean age of 8442 patients in PARADIGM-HF. 93\% people were receiving beta blockers and $60 \%$ were receiving mineral corticoid receptor antagonist, patient had persistent symptoms and signs of heart failure.
The investigators reported a $20 \%$ reduction in the primary outcome of death from cardiovascular causes or hospitalization in patients with heart failure who received sacubitril/valsartan compared with enalapril.

\section{CONCLUSION}

Despite the significant advances in the diagnosis and treatment of heart failure, the disease continues to remain at a higher risk of re-hospitalization, mortality and morbidity worldwide. Inspite of the presence of several drugs such as angiotensin-converting enzyme inhibitors, beta blockers, angiotensin receptor blockers and aldosterone antagonists for HF, patients with the illness continue to have a downward spiral due to the disease progression.

It can conclude that Sacubitril - valsartan an ARNI represents a major therapeutic advance in the avenue of CV disease, particularly HF. This drug has demonstrated an excellent safety profile, and the drug is purported to become a block-buster for the treatment of HF. The therapeutic effect of the drug has been effective in reducing the risk of cardiovascular death and hospitalization among HF patients with reduced ejection fraction.

\section{REFERENCE}

1. Srikanth Yandrapalli, Mohammed Hasan Khan, Yogita Rochlani \& Wilberts Aronow; Sacubitril/Valsartan in cardiovascular disease; Therapeutic Advances in Cardiovascular Disease; June 2018;12(8); 217-231.

2. Arathi A. Inamdar \& Ajinkya C. Inamdar; Heart Failure: Diagnosis, management \& utilization; Journal of Clinical Medicine; Jul 2016; 5(7); 69-82.

3. Ramesh. R. Dargad, Mahesh. R. Prajapathi, Rohit .R. Dargad, Jai. D. Parekh; Sacubitril/valsartan: A novel angiotensin receptor- neprilysin inhibitors; Indian Heart Journal; Jan 2018; 70(1); 102-110.

4. Yandrapalli S, Andries. G, Biswas. M, Khera. $S$; Profile of sacubitril/valsartan in the treatment of heart failure: patient selection \& perspectives; Journal of 
Vascular Health Management; Oct 2017; 13; 368-382.

5. Steven. G. Chrysant, George. S. Chrysant; Sacubitril/Valsartan: a cardiovascular drug with pluripotential actions; Journal of Cardiovascular Diagnosis \& Therapy; May 2018; 8[4]; 549-551.

6. Helena Norberg, Ellinor Bergdahl, Krister Lindmark; Eligibility of sacubitril-valsartan in a real world heart failure population; ESC Heart failure; Apr 2016; 5(17); 13421347.

7. Oriol Iborra-Egea, Carolina GalvezMonton, Antoni Bayes- Genis; Mechanism of action of sacubitril-valsartan on cardiac remodelling, a system biology approach; Biology and Applications; Apr 2017; 12(8); 1105-1108.
8. Michele Senni, Hendrik Streetkerk, Vikas Modgill, Wenchun Zhou, Allen Kaplan; Angioedema in heart failure patients treated with sacubitril-valsartan or enalapril in the PARADIGM-HF study; International journal of cardiology; Dec 2017; 26(4); 118-123.

9. Andries G, Biswas M, Khera S; Profile of sacubitril/valsartan in the treatment of heart failure: patient selection and perspectives; Journal of vascular health and risk management; Oct 2017; 13(4); 369-382.

10. Randall. C. Starling; Sacubitril/valsartan \& the evolution of heart failure care; Cleveland clinic journal of medicine; Oct 2015; 82(10); 702- 704. 